Monatsschrift f. Geburtshülfe u. Gynäkologie 1914;40:I-V

\title{
Contents, Vol. 40, 1914
}

lnhalts -Verzeichnis.

Original-Arfoeiten. Selte

Ahlfeld,F., Puerperale Infektion im Anschlxiß an Retention

von Placentaresten 537

Amann, J. A., Darmruptur durch stumpfe Gewalt bei

Gravidität 633

Anderes, A., Unterbrechung der Schwangerschaft und

Sterilisation auf abdominellem Wege in einer Sitzung 443 B e c k m a n n, W., Über

vorgeschrittene und axisgetragene

Extrauteringravidität 187

Benestad, G., Über die Ernährungsverhältnisse Neu-

geborener 674

B e t k e , H., Die Couveusenbehandlung der Frühgeborenen

und Lebensschwachen

255

B i s c h o ff, C. W., Zxir Differentialdiagnose der Appendicitis

chronica 398

Brattström, E., Ein Fall von viereiigen Vierlingen

nebst einigen Beobachtungen betreff $s$ der Vierhngsgeburten

im allgemeinen

53

Dirks, Marie, G. Marmetschke xmd K r i e b e 1,

Gasphlegmone nach kriminellem Abort $\quad 376$

E d er, A., Zur Kenntnis der Eigenschaften der radioaktiven

Substanzen und ihrer Anwendung 247

Fabricius, Jos., Über ein primäres Karzinom der

Bartholinischen Druse69

F e t z e r, M., Über Spezifität der Abderhaldenschen Ferment-

reaktion 598

Graefe, Gerh., Über Tuberkulose des weiblichen Genital-

apparates im Kindesalter $\quad 448,574$

Hellmuth, Übt die Menstruation einen Einfluß auf die

Hämolyse der Scheidenkeime aus ? . . . 589

Klaatsch, H., Das Problem des menschlichen Hymen.

(Hierzu Taf. I) 332

Malinowsky,M., Tokodynamometrische Untersuchungen

über die Wirkung des Pituitrins auf die Uteruskontrak-

tionen unter der Geburt

$-\mathrm{IV}-$

Seit $\beta$

Martin, A., Der intrauterine Stift 
Nebesky, O., Beitrag zur Kenntnis der Chorioangiome 4201 o w , J., Über die Behandlung der in den früheren Monaten unterbrochenen Extrauterinschwangerschaft 25, 205

Sachs , E., Weitere Erfahrungen mit Pituglandol in der Geburtshülfe. Mit besonderer

Berücksichtigung der Ver-

wendung der intravenösen Injektion 544

Schmauch, G., Ziele und Zwecke einer sachgemäßen

Schilddrüsenbehandlung 224

Schubert, Gotth., Die Behandlung von Tumoren mit

Tumorextrakten 487

Schultze, B. S., Gynâkologie und Psychiatrie 276

Ulesko-Stroganowa, K.. Zur Histogenese der Uterus-

myome387

Vértes, 0., Zur Pathogenese der Eklampsie . · 361,466 Wolff, P., und M. Z a d e , Zur

Diagnose und Prognose

der Nierenveränderungen in der Schwangerschaft . . .639

Tagesfragen.

Zur Strahlentherapie. Von A. Martin 404

Karl Breus $† 307$

Heinrich Fasbender $\dagger 306$

Alfred Hegar $\dagger \quad 331$

F. A. Kehrer $\uparrow \ldots 143$

Karl Paw $\prod \mathrm{k} \uparrow 511,684$

Sammelreferate.

Über die geburtshülflich-gynäkologisehe Literatur in Groß-

britannien und Irland 1913. Von A 1 b a $n$ D o r a $n$ in

Kensington 497

Italienische Literatur über Geburtshülfe und Gynâkologie

1912-1913. Von Paul Hüssy in Basel 75

Bericht über die russische gynäkologische Literatur des Jahres

1913. VonWilhelm B e c k m a $\mathrm{n} \mathrm{n}$ in Petersburg. 100

Brief aus Schweden. Von John Olowin Lund .... 610

Wege und Ziele auf dem Gebiete der inneren Sekretion vom

gynäkologischen Standpunkt. Von Josef Novak in

Wien 280

Die geburtshülflich-gynäkologischen Dissertationen der deut-

schen Universitäten (Winter-Semester 1913/14) . . 112

Seitc

Berichte.

Der VII. Internationale Kongreß für Geburtshülfe und Gynä

kologie 1915142

Tagesnaehrichten und Personalien 143, 308, 511, 686

Beteiligung des Personals der deutschen und österreicbisch-

ungarischen Universitäts- und Akademie-E/8auenkliniken

am Weltkriege 681

Vereins- und Literaturbeilagen. 
Bayerische Gesellschaft für Geburtshülfe und Frauenheilkunde

Budapester Kön. Ärzteverein

688

Gynäkologische Gesellschaft in Breslav.

414

Mittelrheinische Gesellschaft für Geburtshülie vnd Gynäkologie

Münchener gynäkologische Gesellschaft $\quad 618,687$

Niederrheinisch-Westfälische Gesellschaft für Gynäkologie

und Geburtshülfe

165

Nordostdeutsche Gesellschaft für Gynäkologie

Nordwestdeutsche Gesellschaft für Gynäkologie

421

Referate aus geburtshülflich-gynäkologischen Gesellschaften $179,322,432,531,628,695$

Geburtshülflich-gynäkologisehe Mitteilungen aus anderen Ge sellschaften und Vereinen ... 180, 324, 433, 532, 629,

Buchanzeigen 324, 434, 533

Literaturverzeichnis 181, 327, 440, 533, 631, 697

Beriehtigung 186, 511

Sachregister $\quad 701$

Namenregister $\quad 706$ 